\title{
Space-Time Characterization and Collective Motion at Intermediate Energies
}

\author{
Giuseppe Verde \\ GANIL, CEA and IN2P3-CNRS, Caen Cedex, F-14076, France and \\ INFN, Sezione di Catania, I-95123, Italy
}

Received on 10 December, 2006

\begin{abstract}
Shape analyses of two-particle correlation functions are discussed. Two-proton imaging provides information about the volume, the relative contributions between fast and slow emitting sources and the profile of the twoparticle source that can be directly compared to microscopic model simulations. In the case of correlations between complex particles, the role played by collective motion and space-momentum correlations needs special considerations. By means of a semi-quantitative Monte Carlo approach, deuteron-alpha correlation functions measured in $\mathrm{Xe}+\mathrm{Au}$ collisions at $\mathrm{E} / \mathrm{A}=50 \mathrm{MeV}$ are investigated. The comparison reveals the existence of a position-relative momentum correlation that reduces the apparent source size and distorts the line-shape of the correlation function. The developed ideas show how intensity interferometry with complex particles is sensitive not only to the geometry of the system but also to the interplay between collective and thermal motion.
\end{abstract}

Keywords: Two-particle correlation functions; Imaging; Collective motion; Nuclear densities

\section{INTRODUCTION}

Two-particle correlation functions have been extensively used to study the space-time properties of nuclear reactions [1-6]. During an energetic collision between two heavy ions, nuclear matter under extreme conditions of temperature and density is produced. Measuring the properties of the produced nuclear systems represents the only terrestrial means to access the equation of state of nuclear matter. However, heavy-ion collisions are rather complex systems. Particles are emitted by several different sources and a clear understanding of their properties has not yet been achieved. The observed single particle spectra contain contributions from the fast preequilibrium emission, dominating the early stages of the reaction, and the slowly evolving evaporative and secondary decay sources as well. In this context, intensity interferometry techniques have provided powerful tools to disentangle the contributions from different emitting sources and extract information about their properties such as size and lifetimes.

It should be pointed out that the coexistence of very different emission time scales, ranging from pre-equilibrium $(\tau \approx$ $10-50 \mathrm{fm} / \mathrm{c})$ and secondary decays $\left(\tau \approx 10^{3}-10^{5}\right)$ makes the study of such space-time probes of the reaction quite complicated. Two-proton correlation functions have been successfully used to determine the size and the lifetime of particle emission sources $[4,6]$. However, it is expected that different particles are emitted at different times. Thus, extending HBT techniques to particles heavier than protons would represent an important step forward towards fully understanding particle and fragment emission mechanisms and, eventually, their inner link to liquid-gas phase transitions in nuclear matter [7]. On the other hand, one has to keep in mind that the larger mass of complex particles makes them more sensitive to the presence of collective motion, as compared to the case of protons. Indeed, while thermal velocities decrease with mass $\left(v_{t h} \propto m^{-1 / 2}\right)$, collective velocities do not depend on mass.

In this article, we will present some studies that have been recently performed in the context of heavy-ion collisions at intermediate energies. We will focus on the importance of characterizing the space-time extent of the emitting sources by means of detailed analyses of the shape of two-particle correlation functions. The first part of the article is devoted to presenting the advances in the field of imaging techniques applied to angle-averaged two-proton correlation functions. In the second part of the article, attempts to extend shape analyses to correlation functions between complex particles will be discussed. In this context, the role played by collective motion on deuteron-alpha correlation functions measured in $\mathrm{Xe}+\mathrm{Au}$ collisions at $\mathrm{E} / \mathrm{A}=50 \mathrm{MeV}$ will be discussed. This specific case of correlation functions between complex particles can be considered as a guideline to understand how the effects of collective flow in nuclear reactions can strongly affect shape analyses and must be properly taken into account in order to extract reliable space-time source extents.

\section{TWO-PROTON CORRELATION FUNCTIONS AND SOURCE FUNCTIONS}

Given two protons with individual momenta $\vec{p}_{1}$ and $\vec{p}_{2}$, relative momentum $q$, and relative space-time separation, $\vec{r}$, the angle-averaged correlation function, $1+R(q)$, can be defined as:

$$
1+R(q)=4 \pi \int S(r) K(r, q) r^{2} d r
$$

usually referred to as Koonin-Pratt equation $[2,8]$. The source function, $S(r)$, is defined as the probability distribution for emitting a pair of protons with a space-time separation $r$ in their center of mass frame. This separation is calculated at the instant of time when the last one of the two protons is emitted. The interplay between the Pauli exclusion principle and the final state Coulomb and nuclear interactions is contained in the angle-averaged kernel, $K(q, r)$, that can be calculated from the two-particle radial wave function. The KooninPratt equation is used to extract information about the emitting two-particle source function, $S(r)$. The angle-averaged source function contains a space-time ambiguity that can be resolved by means of directional correlation studies $[8,9]$. However, 
directional analyses are successful when the emission time scales and not too long. In a nuclear reaction between heavy ions, protons are expected to be produced by different mechanisms and the $\mathrm{p}-\mathrm{p}$ correlation function will reflect the properties of different emitting sources.

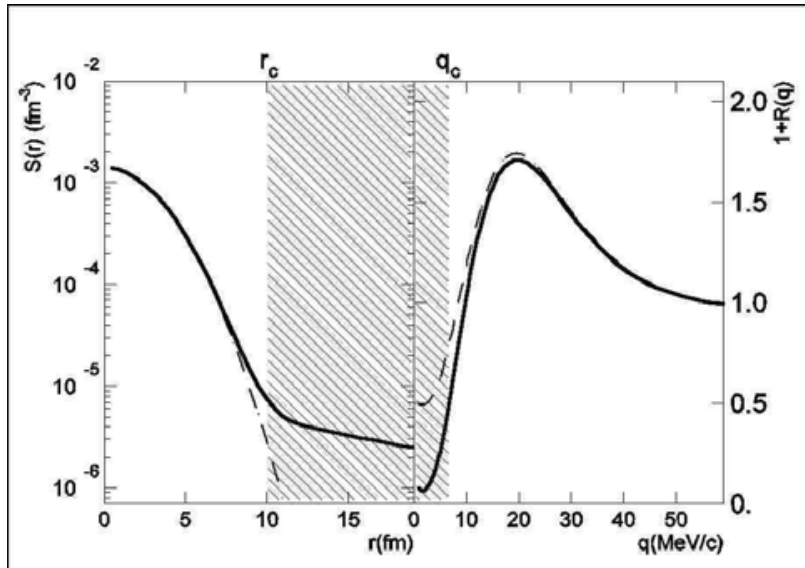

FIG. 1: Left panel: The thick line indicates a typical two-proton source function for intermediate energy collisions. The dashed line represents the short range contribution from short lifetime preequilibrium emission. Right panel: corresponding correlation functions calculated with Eq. (1).

The thick solid line on the left panel of Fig. 1 shows a typical profile for a two-proton emitting source [10]. A short range contribution, at small $r$-values, peaks at $r=0$ and localizes within a small range in $r$-space. This portion is dominated by pairs generated by fast pre-equilibrium emissions in the earlier stages of the reaction. A significant number of protons are known to be produced also by long lifetime evaporative emissions and from secondary decays of the heavier fragments. These protons are expected to contribute mainly in the long tail of the source function at large relative spatial distances. The corresponding proton-proton correlation function from this two-component source is represented on the right panel of Fig. 1 by the thick solid line. The prominent peak at $20 \mathrm{MeV} / \mathrm{c}$ is due to the attractive nature of the short range nuclear interaction between the protons. This type of interaction dominates proton pairs at small relative distances in the source function. However, at large $r$-values, the Coulomb force and the Pauli exclusion principle are the dominant factors. Thus, the portion of $S(r)$ contained in its tail is expected to contribute mainly at the low relative momentum region of $1+R(q)$ (shaded regions in Fig. 1). In order to show this, we represent the short range part of $S(r)$ with a more localized source (dashed line on the left panel of Fig. 1). Its integral over all space, $\lambda$, is less than unity, due to the suppression in the tail. As expected, the correspondent correlation function (dashed line in the right panel) is strongly affected in the region $q=0-10 \mathrm{MeV} / \mathrm{c}$. Unfortunately, this region at low relative momenta (shaded area) is normally not accessible. In particular, the presence of experimental threshold in the data, $q_{c}$, (commonly ranging between 5 and $15 \mathrm{MeV} / \mathrm{c}$ ), suggests that the correlation function probes only a portion of the source $S(r)$, inside a certain cutoff relative distance, i.e. $r<r_{c}$. In such a scenario, the commonly adopted picture of using the height of the peak at $20 \mathrm{MeV} / \mathrm{c}$ to extract the size of the emitting source has to be abandoned. This is shown on left panel of Fig. 2 where three correlation functions corresponding to sources with different sizes display the same values at $\mathrm{q}=20 \mathrm{MeV} / \mathrm{c}$. The $r_{1 / 2}$ values of the source profiles are used as a measure of their space-time extent. Their integrals, $\lambda=0.38,0.61$ and 1.0 , reflect different long range tail suppressions from the original sources. It is evident that the shape, not the height, of the peak at $20 \mathrm{MeV} / \mathrm{c}$ reflects the size of the fast pre-equilibrium proton source. This is best shown on the right panel of Fig. 2 that represents the correlation between the size $\left(r_{1 / 2}\right.$-value on the lower scale and rms-radius on the upper scale) of the fast portion of the emitting source and the FWHM width, $\Delta q_{F W H M}$, of the peak in the correlation function. $\Delta q_{F W H M}$ is linearly correlated to the size of the emitting source: more extended sources produce a more narrow peak at $20 \mathrm{MeV} / \mathrm{c}$. Furthermore, the correlation between width of the peak and size of the source is completely independent of the $\lambda$-values, i.e. the relative contributions between fast and slow proton emissions [10].

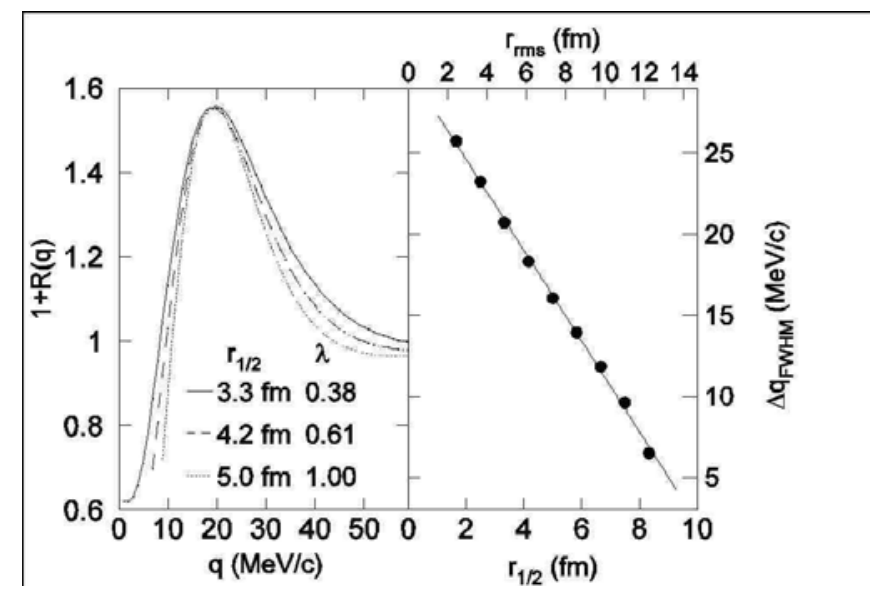

FIG. 2: Left panel: correlation functions for Gaussian sources with different $\lambda$-values and radii. Right panel: the relation between the width of the resonance peak at $20 \mathrm{MeV} / \mathrm{c}$ and the $r m s$-radii (upper scale), and half radii (lower scale).

\section{IMAGING TWO-PROTON CORRELATION FUNCTIONS}

The above considerations show that a study of the whole shape of the correlation function is required to extract the correct size of the emitting source function. To utilize all the information measured in the correlation function including the regions outside the resonance peak at $q=20 \mathrm{MeV} / \mathrm{c}$, a model independent imaging technique has been used $[11,12]$. The basic idea behind it is to extract the source function $S(r)$ by a numerical inversion of the Koonin-Pratt equation, Eq. (1), 
without any a priori assumption about its profile. All the data points of the correlation function deviating from unity, are used to obtain information about the source function.

Combining the flexibility of the imaging approach with our qualitative understanding of the link between the main physical properties of the source and the global features of the correlation function, we have analyzed the momentum dependence of two-proton correlations measured in ${ }^{14} \mathrm{~N}+{ }^{197} \mathrm{Au}$ reactions at $75 \mathrm{MeV} / \mathrm{u}$ [6]. Details of the experiment can be found in [13]. The data points on Fig. (3) show the protonproton correlation functions measured for three bins of total momentum, $P_{\text {sum }}$, in the lab frame, $P_{\text {sum }}=840-1230,450-780$ and 270-390 MeV/c.

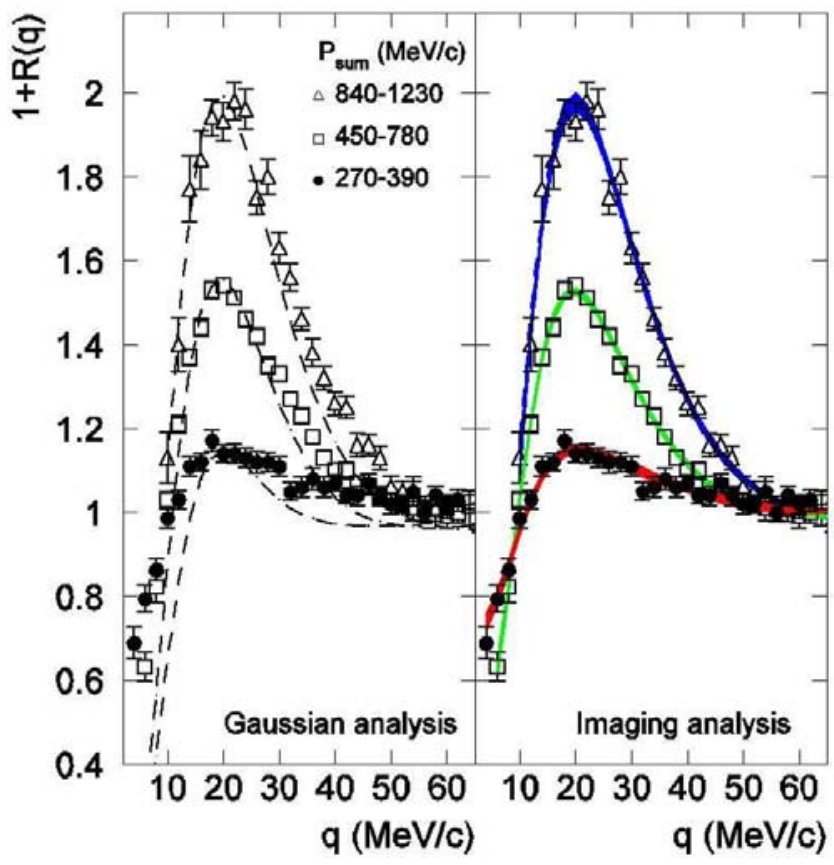

FIG. 3: (Color online) Gaussian analysis (left panel) and Imaging analysis (right panel) of two-proton correlation functions measured in ${ }^{14} \mathrm{~N}+{ }^{197} \mathrm{Au}$ collisions at $\mathrm{E} / \mathrm{A}=30 \mathrm{MeV}$. See text for details [10].

The thick curves on the right panel of Fig. 3 show the bestfits of the experimental data with the imaging approach [10]. All the data points deviating from unity are reproduced by the numerical inversion of Eq. (1). As a comparison, the left panel of Fig. 3 shows the results of a more common onedimensional analysis approach[13, 14]. Indeed, the dashed lines correspond to a best fit of the experimental data performed by using Gaussian sources with the width used as the only free parameter. The failure of such approach clearly demonstrates the non-Gaussian nature of the sources. A correct extraction of the source function requires a detailed description of the whole shape of the correlation function. This goal is achieved successfully by the imaging technique and the resulting emitting sources are shown by the thick lines on Fig. 4 [10]. The dashed lines refer to the Gaussian sources used to fit the data on the left panel of Fig. 3. It is clearly

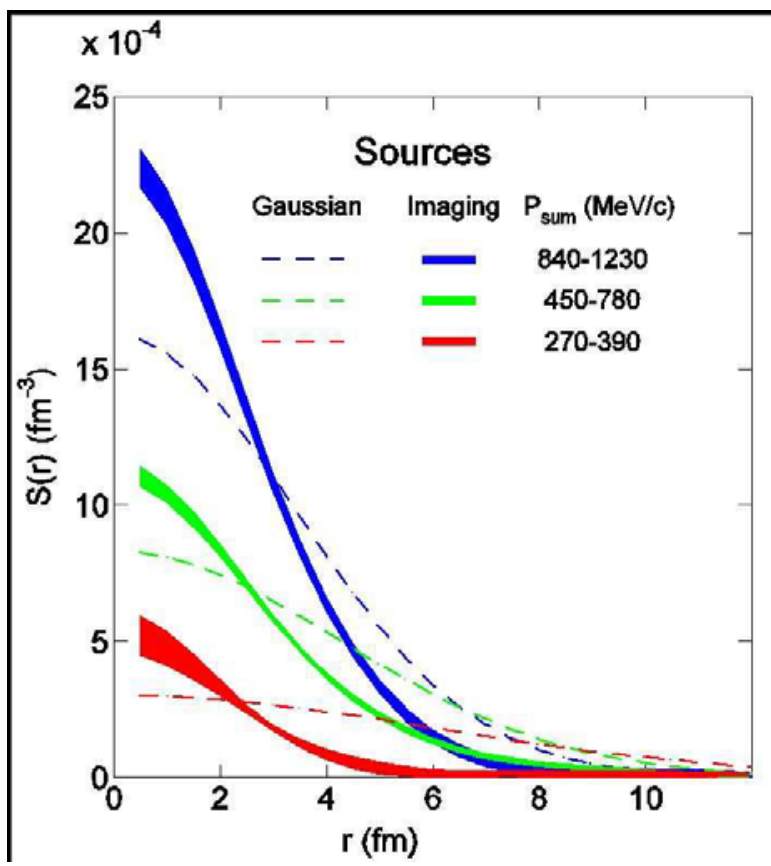

FIG. 4: (Color online) Solid thick lines: imaged sources. Dashed lines: Gaussian sources. See text for details [10].

seen that without a detailed shape analysis of the correlation function, the emitting sources are not correctly extracted.

As we outlined in section 2, the imaged sources, represented in Fig. 4 refer to the short range portion, dominated by fast pre-equilibrium emissions. Their sizes, quantified by their $r_{1 / 2}$ values are $2.44 \pm 0.4 \mathrm{fm}, 3.13 \pm 0.14 \mathrm{fm}$ and $2.93 \pm 0.15$ $\mathrm{fm}$, for $P_{\text {sum }}=840-1230,450-780$, and 270-390 MeV/c, respectively. The total momentum dependence of these sizes is shown in the left panel of Fig. 5. Consistent with previous analysis, the widths of the Gaussian source (open circles) decrease strongly with increasing total momentum of the two protons. However, these sizes are not correct because the Gaussian source does not fit the experimental data (see Fig. 3). The sizes extracted by imaging (solid points) do not show a strong dependence on the total momenta of the pairs. This weak sensitivity to $P_{\text {sum }}$ of the short range sizes of the imaged sources, refer mainly to a property of the small localized emitting region in space-time corresponding to preequilibrium emissions. Proton pairs produced by long lifetime emission mechanisms, such as evaporation and secondary decays, mainly contribute at large relative distances $r$, outside the imaged region, due to the experimental thresholds in the measured correlation functions.

To quantify the contributions from the short range source, we calculate the integrals, $\lambda$, of the imaged sources from 0 to a value of $r$ where the source function itself it still significant. The deviations of this integral from unity, $1-\lambda$, are the contribution from the long-lived sources. The analysis suggests that about 40,55 and $90 \%$ of proton pairs, for $\mathrm{P}=840$ $1230,450-780$ and $270-390 \mathrm{MeV} / \mathrm{c}$, respectively, are missing from the imaged region. These protons are produced mainly 


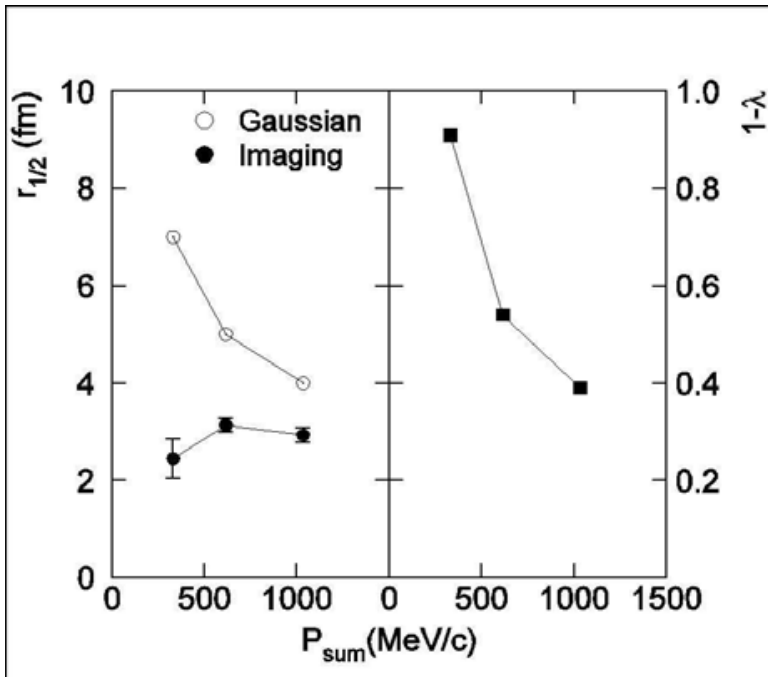

FIG. 5: Source size (left panel) and contributions from long-lifetime proton emission (right panel) as a function of total momentum, $P_{\text {sum }}$.

from evaporative processes and secondary decays, typical in the later stages of the reactions. All the strong total momentum dependence observed in the correlation data is reflected in contributions from long-lifetime emissions (as shown on the right panel of Fig. 5, rather than in the size of the imaged sources.

These results demonstrate clearly that extracting the size of the emitting source requires a detailed shape analysis of the correlation function. This consideration can be easily extended to correlation functions constructed with particles heavier than protons. Failures in reproducing the line-shape of the correlation function leads to large inaccuracies as far as the volume of the source is concerned.

\section{PROBING TRANSPORT MODELS BY IMAGING TWO-PROTON CORRELATIONS}

The source extent shown on Fig. 5 represents low order information about the source itself. Of course, the whole profile of the source extracted by means of the imaging technique contains much more information. This profile can indeed be used to constrain certain features of microscopic reaction models. Within the context of reaction models it is possible to calculate the two-proton emitting source directly from the single particle emission functions $[15,16]$. Comparisons to the source profiles extracted from the experimental data by means of the imaging technique allow one to perform more stringent tests of transport properties of nuclear matter.

In an attempt to constrain BUU transport model predictions, two-proton correlation functions measured in central $\mathrm{Ar}+\mathrm{Ac}$ at $\mathrm{E} / \mathrm{A}=80,120$ and $160 \mathrm{MeV}$ have been imaged $[17,18]$. The main difficulty in performing this kind of analysis stems from the fact that BUU does not contain any fragment formation mechanisms. Therefore, BUU simulations are more suited to describe the early dynamical stage of the reaction. In the previous section we have explained how imaging provides the profile of the dynamical stage and a quantitative estimate of the relative contributions from the fast pre-equilibrium source and the slow secondary decay emitting source. Based on such considerations, the imaging analyses of the experimental data in Ref. [18] was performed to extract both the profile of the dynamical source and also a normalization constant that can be identified with the $\lambda$ parameter in Fig. 5. This normalization factor was used to scale the BUU two-proton source functions to better compare to the imaged source profiles.

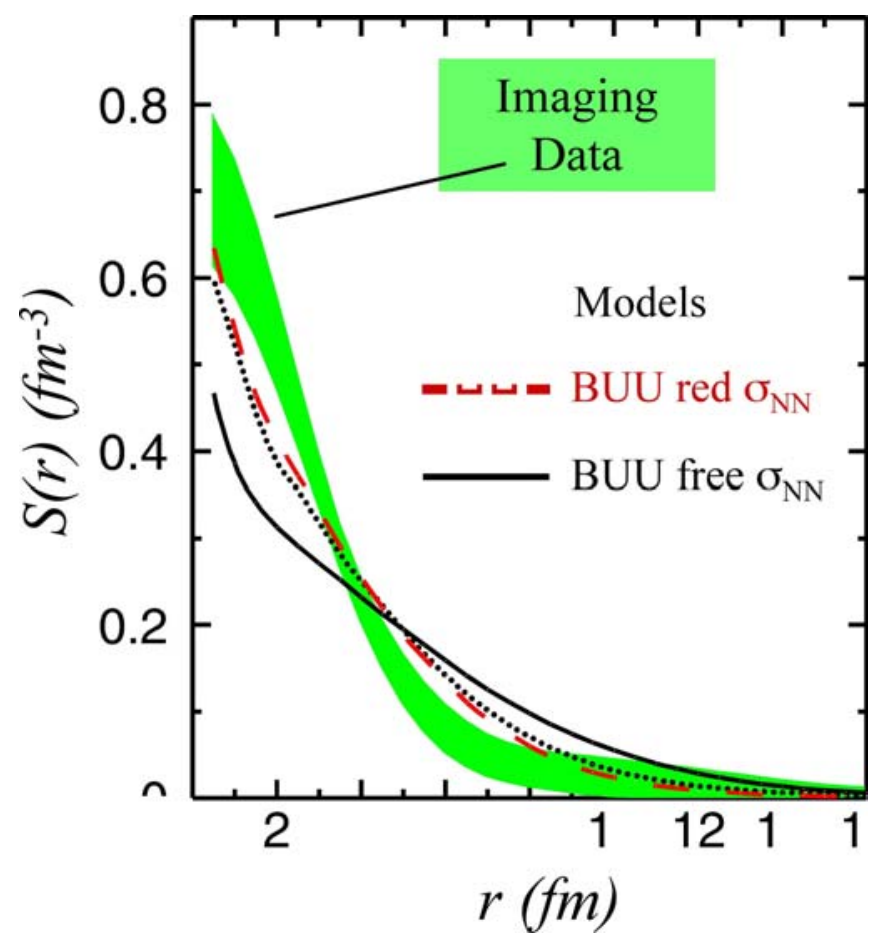

FIG. 6: (Color online) Two-proton source function in central $\mathrm{Ar}+\mathrm{Sc}$ collisions at $\mathrm{E} / \mathrm{A}=80 \mathrm{MeV}$. Shaded area: results from imaging; Lines: BUU simulations with free (solid) and reduced (dotted and dashed lines) nucleon-nucleon cross section.

Fig. 6 refers the case of $\mathrm{Ar}+\mathrm{Sc}$ collisions at $\mathrm{E} / \mathrm{A}=80 \mathrm{MeV}$. A comparison between imaged sources (shaded area) and calculated sources (lines) using the BUU transport model [18] is shown. This comparison shows that source profiles obtained from imaging can be used to probe the nucleon-nucleon cross section. The overall features of Fig. 6 show that for free NN cross sections, BUU predicts two-particle sources that are too extended (solid line). This correspondence between the size of the source and the width of the correlation peak was known in early studies with proton-proton HBT $[14,17]$. A reduction of the in-medium NN cross section in BUU simulations produces sources that are in reasonable agreement with the imaged ones (dashed line). This conclusion based on a detailed imaging analysis of the measured correlation functions is different from the one presented in Ref. [17] where the same set of data was analyzed and where the best agreement between BUU calculations and correlation function data was attained 
when free cross section values were used. The results on the cross section reductions reported here are consistent with the conclusions deduced in Ref. [19] from the analysis of flow data. Despite these successes at $\mathrm{E} / \mathrm{A}=80 \mathrm{MeV}$, at higher energies, $\mathrm{E} / \mathrm{A}=160 \mathrm{MeV}$, the shape of the two-proton source from imaging exhibits a core+tail behavior that is not reproduced by transport model simulations. The shape of the BUU twoproton source appears incorrect, no matter what cross section is used.

Regardless of the moderate success of imaging-vs-BUU analyses, it should be pointed out that detailed shape studies of two proton correlation functions provide not only reliable space-time extents but also higher order information, such as the source emitting profile, that can be considered a useful probe to test the validity of microscopic models.

Motivated by these results and by the importance of shape studies, one can attempt to apply imaging technique to complex particle correlations. However, other complications can play a relevant role and the task becomes difficult. In the next section, shape analyses of deuteron-alpha correlation functions are discussed in the context of the effects induced by space-momentum correlations on HBT studies.

\section{POSITION-RELATIVE MOMENTUM CORRELATIONS}

Particles produced during heavy-ion collisions move with velocities consisting of a thermal and a collective component. The collective component is commonly described with position dependent velocity fields. In central collisions the existence of radial expansion has been observed and the corresponding collective velocities have been extensively parameterized with a self-similar field, $\vec{v}(\vec{r}) \propto \vec{r} / R$, where $R$ is the radius of the emitting source. In the case of intensity interferometry analyses, these collective velocity fields are known to induce "position-total momentum correlations" $(\vec{r}-\vec{P}$, where $\vec{P}=\vec{p}_{1}+\vec{p}_{2}$ ) [4-6]. As a consequence, smaller source sizes are expected to be observed when particles pairs with higher velocities are selected [3, 4]. This contribution shows other features that can emerge from space-momentum correlations induced by collective motion and that can severely affect HBT analyses with massive particles. We will focus on the case of deuteron-alpha correlations.

We simulate the emission of $d-\alpha$ pairs by means of a simple Monte Carlo model where the system is described as a sphere with radius $R$ and temperature $T$. The source is assumed to expand radially with a self-similar velocity field, $\vec{v}(\vec{r})=\beta \vec{r} / R$. For each emitted pair the relative separation, $r$, and the momentum of relative motion, $q=\mu v_{\text {rel }}$, are recorded. Fig. 7 shows a color scatter plot of $r$ vs $q$ in the case of a pure thermal source, $\beta=0$ (upper panel), and in the case of a radially expanding source with $\beta=0.2$ (bottom panel). It is clear that collective motion induces a correlation between relative position and relative momentum.

What are the effects induced by these "relative momentumposition" correlations on HBT analyses? The main effects consist of: a) an apparent reduction of the source size and b) a distortion in the line shape of the corresponding corre-

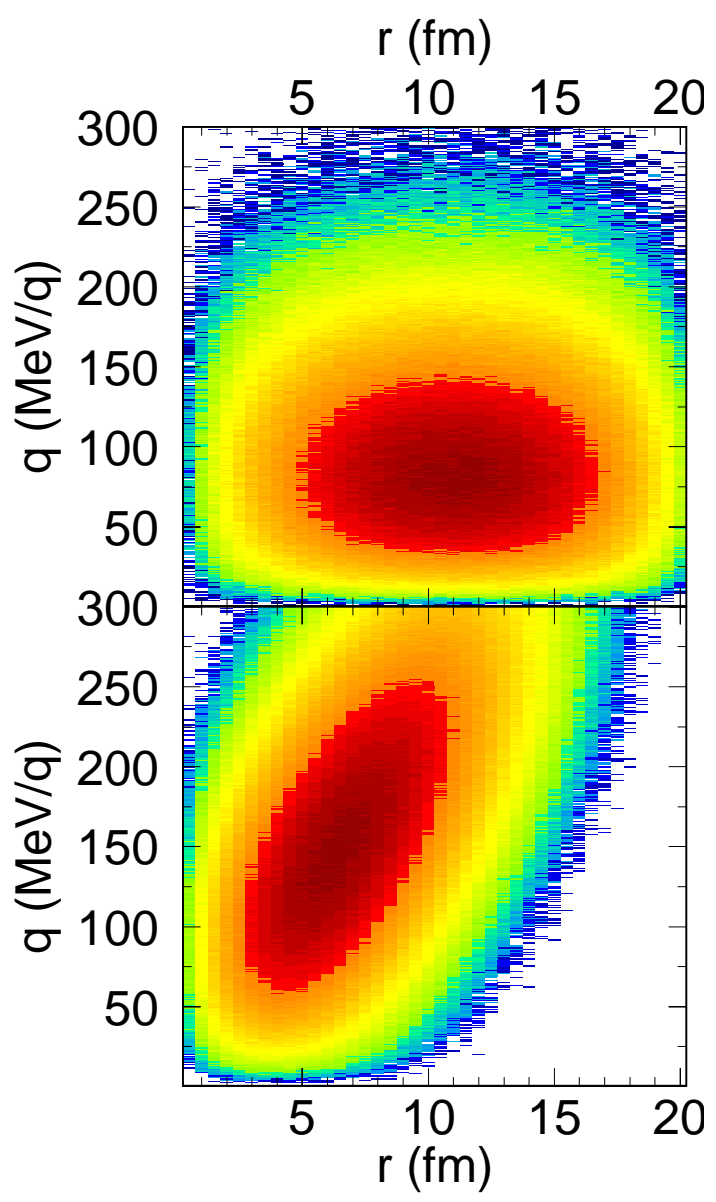

FIG. 7: (Color online) Relative momentum-position correlations in the case of pure thermal sources with $\beta=0$ (top panel) and in the case of thermal and expanding sources with $\beta=0.2$ (bottom panel). See text for details.

lation function. Both effects change drastically the analysis techniques for complex particle correlation functions as well as the space-time information that one can extract from them [20].

The reduction of the apparent source size can be easily explained by the scatter plot shown in the bottom panel of Fig. 7. In the presence of space-momentum correlations, small spatial extents of the source are associated to correspondingly small relative momenta. Measurements of correlation functions normally extend to finite relative momentum ranges, $0 \leq q \leq q_{\max }$, with $q_{\max }$ of the order of few hundreds of $\mathrm{MeV} / \mathrm{c}$ 's. As a consequence, the probed region of the emitting source is reduced as compared to the case of a pure thermal source where any relative momentum regions probe particles emitted by the whole source spatial extent.

The other important effect of position-relative momentum correlations consists of a distortion in the line-shape of the correlation function. This effect is described in the next section by showing a study of deuteron-alpha correlation functions measured a heavy-ion collision experiment at intermediate energies. 


\section{LINE-SHAPE DISTORTIONS IN $d-\alpha$ CORRELATION FUNCTIONS}

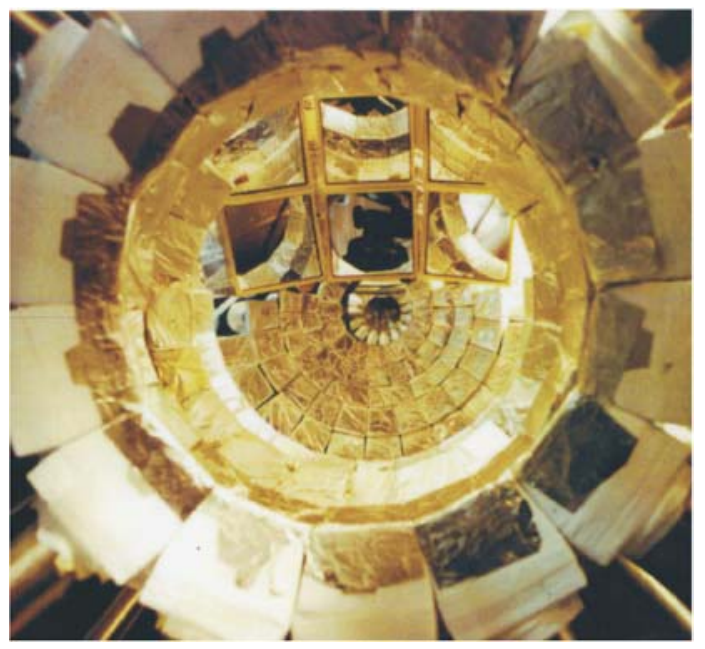

FIG. 8: (Color online) The LASSA array located inside the Miniball $4 \pi$ detector at the NSCL-MSU.

Deuteron-alpha correlation functions were measured in quasi-central $\mathrm{Xe}+\mathrm{Au}$ collisions at $\mathrm{E} / \mathrm{A}=50 \mathrm{MeV}$. The experiment was performed at the K1200 cyclotron of the National Superconducting Cyclotron Laboratory at Michigan State University. Isotopically resolved particles with $1 \leq \mathrm{Z} \leq 10$ were detected with nine telescopes of the Large Area Silicon Strip Array (LASSA). Details about the LASSA array can be found in Refs. [21]. Impact parameter selection was performed with the Miniball/Miniwall arrays [22] by means of the total multiplicity of detected charged particles. Fig. 9 shows the measured d- $\alpha$ correlation function (data points). The correlation function is determined as the ratio between coincidence two-particle yields and uncorrelated two-particle yields constructed by means of the event mixing techniques [23].

The anticorrelation at small relative momentum, $q<30$ $\mathrm{MeV} / \mathrm{c}$, is due to the mutual Coulomb repulsion. The sharp peak at relative momentum $\mathrm{q}=42 \mathrm{MeV} / \mathrm{c}$ corresponds the first excited states of ${ }^{6} \mathrm{Li}$ at $\mathrm{E}^{*}\left({ }^{6} \mathrm{Li}\right)=2.186 \mathrm{MeV}$ state, while the broad peak around $84 \mathrm{MeV} / \mathrm{c}$ stems mainly from the resonance at $\mathrm{E}^{*}\left({ }^{6} \mathrm{Li}\right)=4.31 \mathrm{MeV}$. Both peaks are resolved with high resolution by the LASSA array. Theoretically, the angle-averaged correlation function is commonly calculated using the angle-averaged Koonin-Pratt equation 1 . The angleaveraged kernel $K(q, r)$ is calculated from the radial part of the two-particle scattering relative wave function. The source function, $S(r)$, is defined as the probability of emitting two correlated particles separated by a distance $r$ when the last of the two particles is emitted. Due to the presence of collective motion, one can encounter "relative momentum-position" correlations, as it was shown in Fig. 7, that make the source function in Eq. 1 both $r$ - and $q$-dependent, e.g. $S=S(r, q)$.

By means of the Monte Carlo model described in the previous section, we simulate two-particle source functions, $S(r, q)$,

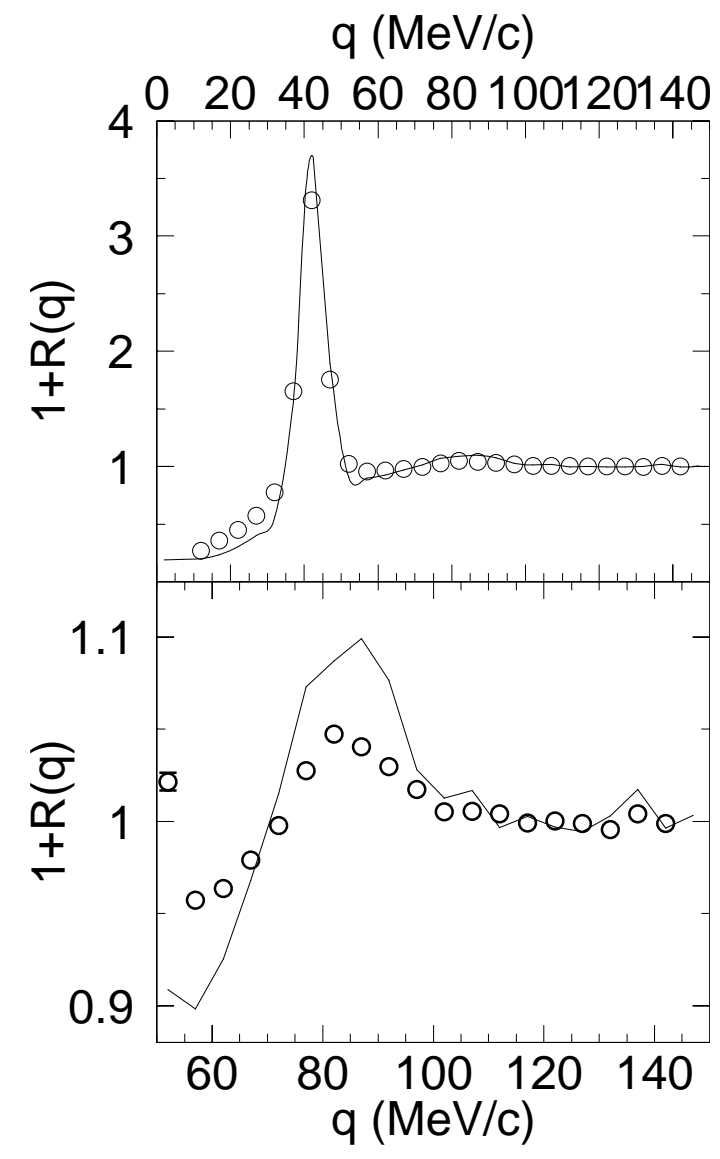

FIG. 9: Data points: Deuteron-alpha correlation functions measured in quasi-central $\mathrm{Xe}+\mathrm{Au}$ collisions at $\mathrm{E} / \mathrm{A}=50 \mathrm{MeV}$. Solid line: Simulated d- $\alpha$ correlations for purely thermal spherical sources with $\mathrm{R}=10.5 \mathrm{fm}$ and temperature $\mathrm{T}=4 \mathrm{MeV}$.

corresponding to a system evaporating at temperature $T$ while undergoing collective motion. The simulated sources are then used in Eq. (1) to calculate the correlation function. The solid lines on Fig. 9 corresponds to a spherical source of radius $\mathrm{R}=10.5 \mathrm{fm}$ and with collective motion turned off, $\beta=0$. The radius of the source is determined from a best-fit of the shape of the second peak at $84 \mathrm{MeV} / \mathrm{c}$ [10]. It is clearly seen that a pure thermal source cannot reproduce the shape of the experimental data. In particular, the heights of the peaks at 42 and $84 \mathrm{MeV} / \mathrm{c}$ cannot be reproduced simultaneously by a pure thermal source. If one reproduces the height of the first peak, the height of the second peak remains overpredicted. The data seem to contain a distortion that cannot be easily resolved. Similar distortions in $d-\alpha$ correlation functions were already observed previously [24]. It is clear that one needs to solve such discrepancies in order to extract reliable size and density information from $d-\alpha$ correlation functions. The predicted "relative momentum-position" correlations observed in Fig. 7 (bottom panel) can provide an explanation for such difficulties. In particular, one needs to take carefully into account the effects of collective motion on those regions of the correlation function that are dominated by nuclear resonances. The 
peaks at q $42 \mathrm{MeV} / \mathrm{c}$ and $\mathrm{q} 84 \mathrm{MeV} / \mathrm{c}$, being generated by the short range mutual d- $\alpha$ nuclear interaction, are mostly populated by d- $\alpha$ pairs emitted from nearly the same location in space with relative distances, $r \approx 0$. Therefore, the deuteron and the alpha particles are characterized by almost the same collective velocity. When calculating their relative momentum in these regions $\mathrm{q}=42 \mathrm{MeV} / \mathrm{c}$ and $84 \mathrm{MeV} / \mathrm{c}$, the collective components cancel out. On the other hand, the mixed event background, used to construct the uncorrelated background in the correlation function includes: a) particles that come from two different events in which the collective velocity fields are completely different; b) particles that are emitted even from very different space locations in the system. Consequently, deuterons and alphas mixed from different events can have very different collective velocity components. Their relative velocity will be on average larger than the one of coincident $d-\alpha$ pairs. The difference between coincidence and uncorrelated spectra induces a distortion in the shape of the correlation function. The distortion induces a sort of exponential decline in the height of the resonant peaks with increasing relative energy. This effect seem to qualitatively explain the problems observed in Fig. 9 where the height of the second peak appears to be attenuated as compared to a situation where collective motion does not exist.

Stimulated by the described considerations, we have compared the experimental data to simulations performed with our Monte Carlo calculations by adding collective motion. In similar reaction systems, a presence of a collective expansion of about $2 \mathrm{MeV}$ per nucleon have been observed by studying fragment kinetic energy spectra $[25,26]$. We included such an amount of collective motion in our Monte Carlo simulations by choosing a radial expansion parameter, $\beta=0.1$. We also assume the emitting system to be characterized by an ellipsoidal shape with axis, $R_{z}=2 \cdot R_{x}=2 \cdot R_{y}$, where $z$ is the direction of the beam axis. This deformation of the system is intended to schematically simulate emissions from projectilelike and target-like sources in non-central collision events. In Fig. 10 the results of the simulations are shown in the region corresponding to the second peak at $84 \mathrm{MeV} / \mathrm{c}$. The solid line corresponds to the correlation function obtained with collective motion and for an ellipsoidal source with $R_{z}=16 \mathrm{fm}, R_{x}=8$ $\mathrm{fm}$ and $R_{y}=8 \mathrm{fm}$. While the radial flow parameter, $\beta$, has been fixed to the value that would be more consistent with the existing literature, the radii of the ellipsoidal source have obtained by minimizing the chi-squared between data and simulations. The dotted line correspond to the pure thermal source that was already shown to fail in reproducing the shape of the correlation function. The correlation functions shown in Fig. 1 are all normalized to reproduce the height of the first peak at 42 $\mathrm{MeV} / \mathrm{c}$. It is clearly observed that including collective motion modifies the line shape of the correlation function improving the comparison to experimental data. The height of the second peak at $84 \mathrm{MeV} / \mathrm{c}$ is attenuated due to the interplay between thermal and collective motion in the event mixing background described earlier. Furthermore, the effects of position-relative momentum correlations on the source size induces also a distortion on the Coulomb part of the deuteron-alpha correlation function. This further distortion is observed in the valley be- tween the two peaks, at $\mathrm{q}=50-70 \mathrm{MeV} / \mathrm{c}$ where the comparison to experimental data improves when collective motion is turned on.

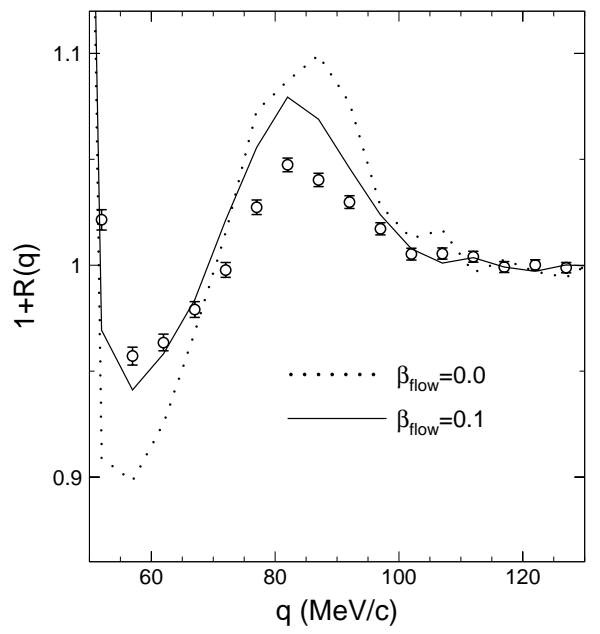

FIG. 10: Expanded view of the peak at $\mathrm{q}=84 \mathrm{MeV} / \mathrm{c}$ in the $\mathrm{d}-\alpha$ correlation function. Open dots: experimental data. Dotted line: pure thermal source. Solid line: emitting source with collective motion, $\beta=0.1$ and elliptical shape with $R_{z}=16 \mathrm{fm}, R_{x}=8 \mathrm{fm}$ and $R_{y}=8 \mathrm{fm}$ (see text).

Even if the Monte Carlo calculations described in this article must be considered only a schematic representation of particle emission properties, they are capable of providing a semi-quantitative description of the data. The distortions in the line-shape of the $\mathrm{d}-\alpha$ correlation function are qualitatively predicted and the parameters used in the model are close to those that have been more precisely determined in other experiments. Assuming that the emitting system contains about $80 \%$ of the total mass of the colliding nuclei, consistent with Refs. $[25,26]$, the density of the d- $\alpha$ source can be estimated in the range $\rho / \rho_{0} \approx 0.1-0.4$, which is reasonably close to density values expected in these reactions and used as input to statistical multifragmentation models.

Better density determination can be performed with improved collective motion models. The simple Monte Carlo approach used here to deuteron and alpha emission represents an oversimplification and it is meant to show what the effects of "relative momentum-position" correlations are on the study of correlation functions between complex particles. On the other hand, collective motion can be determined and constrained most effectively with high precision measurements using modern $4 \pi$ detector designs with high granularity. This would allow a better reconstruction of impact parameter, reaction plans and collective component of particle velocities.

The inclusive analysis shown in Fig. 10 certainly contains contributions from several emitting sources. Gating on total momentum of the emitted particles can certainly improve source selectivity. In this respect, determinations of densities as a function of particle pair velocities are the required tool to 
access the dynamical evolution of source densities during the reaction.

\section{CONCLUSIONS}

By means of a schematic Monte Carlo approach, positionrelative momentum correlations are predicted in the study of intensity interferometry with complex particles interacting with short ranged nuclear forces. The induced effects include an apparent source size reduction and a distortion of the line shape of the correlation function. These distortions are studied in the specific case of deuteron-alpha correlation functions, and used to extract source size information from experimental data collected in the reaction $\mathrm{Xe}+\mathrm{Au}$ at $\mathrm{E} / \mathrm{A}=50 \mathrm{MeV}$. The proposed ideas provide a semi-quantitative explanation of the long-standing problem of the shape of $d-\alpha$ correlation functions. By making reasonable assumptions about collective motion and temperature in the emitting system, we improve our understanding of these complex particle correlations and extract source size and densities that are reasonably close to expectations for the studied systems.

These results can play a key role in the study of correlation functions built with massive particles at all incident energies. The effects of collective motion are indeed more pronounced when heavier particles are considered, due to the decreasing magnitude of thermal velocity. Detailed simultaneous analyses of HBT observables and collective motion are necessary in order to complex particles to extract space-time information about nuclear reactions.
[1] G. Goldhaber, S. Goldhaber, W. Lee, and A. Pais, Phys. Rev. 120, 300 (1960).

[2] S. E. Koonin, Phys. Lett. B 70, 43 (1977).

[3] W. Bauer, C. K. Gelbke, and S. Pratt, Ann. Rev. Nucl. Part. Sci. 42, 77 (1992).

[4] D. H. Boal, C.K. Gelbke, and B. K. Jennings, Rev. Mod. Phys. 62, $553(1990)$

[5] U. Heinz and B. V. Jacak, Ann. Rev. Nucl. Part. Sci. 49, 529 (1999).

[6] M. A. Lisa, S. Pratt, R. Soltz, and U. Wiedemann, Ann. Rev. Nucl. Part. Sci. 55, 357 (2005).

[7] J. Pochodzalla et al., Phys. Rev. Lett. 75, 1040 (1995)

[8] S. Pratt and M. B. Tsang, Phys. Rev. C 36, 2390 (1987).

[9] M. A. Lisa et al., Phys. Rev. Lett. 71, 2863 (1993).

[10] G. Verde et al., Phys. Rev. C 65, 054609 (2002).

[11] D. A. Brown and P. Danielewicz, Phys. Lett. B 398, 252 (1997).

[12] D. A. Brown and P. Danielewicz, Phys. Rev. C 64, 014902
(2001).

[13] W. G. Gong et al., Phys. Rev. C 43, 1804 (1991), and references therein.

[14] W. G. Gong et al., Phys. Rev. C 43, 781 (1991).

[15] W. G. Gong et al., Phys. Rev. Lett. 65, 2114 (1990).

[16] G.J. Kunde et al., Phys. Rev. Lett. 70, 2545 (1993).

[17] D. O. Handzy et al., Phys. Rev. C 50, 858 (1994).

[18] G. Verde et al., Phys. Rev. C 67, 034606 (2003).

[19] G. D. Westfall et al., Phys. Rev. Lett. 71, 1986 (1993).

[20] G. Verde et al., submitted for publication on Physics Letters B.

[21] B. Davin et al., Nucl. Instr. and Meth. A 473, 302 (2001).

[22] R.T. de Souza et al., Nucl. Inst. Meth. A 295, 109 (1990).

[23] M. A. Lisa et al., Phys. Rev. C 44, 2865 (1991).

[24] C. B. Chitwood et al., Phys. Lett. B 172, 27 (1986).

[25] N. Marie et al., Phys. Lett. B 391, 15 (1997).

[26] C. Williams et al., Phys. Rev. C 55, R2132 (1997). 